\title{
Bone Marrow Microenvironment in Multiple Myeloma Progression
}

\author{
S. Manier, ${ }^{1,2}$ A. Sacco, ${ }^{1}$ X. Leleu, ${ }^{2}$ I. M. Ghobrial, ${ }^{1}$ and A. M. Roccaro $^{1}$ \\ ${ }^{1}$ Dana-Farber Cancer Institute, Department of Medical Oncology, Harvard Medical School, 450 Brookline Avnue, \\ HIM 246, Boston, MA 02215, USA \\ ${ }^{2}$ Service des Maladies du Sang, CHRU de Lille, 59000 Lille, France
}

Correspondence should be addressed to A. M. Roccaro, aldo_roccaro@dfci.harvard.edu

Received 3 August 2012; Accepted 18 September 2012

Academic Editor: Sue-Hwa Lin

Copyright () 2012 S. Manier et al. This is an open access article distributed under the Creative Commons Attribution License, which permits unrestricted use, distribution, and reproduction in any medium, provided the original work is properly cited.

\begin{abstract}
Substantial advances have been made in understanding the biology of multiple myeloma (MM) through the study of the bone marrow (BM) microenvironment. Indeed, the BM niche appears to play an important role in differentiation, migration, proliferation, survival, and drug resistance of the malignant plasma cells. The BM niche is composed of a cellular compartment (stromal cells, osteoblasts, osteoclasts, endothelial cells, and immune cells) and a noncellular compartment including the extracellular matrix (ECM) and the liquid milieu (cytokines, growth factors, and chemokines). In this paper we discuss how the interaction between the malignant plasma cell and the BM microenvironment allowed myeloma progression through cell homing and the new concept of premetastatic niche.
\end{abstract}

\section{Introduction}

Multiple myeloma (MM) is a hematologic malignancy characterized by the accumulation of monoclonal plasma cells in the bone marrow (BM), over 10\% by definition [1]. In almost all cases, MM is preceded by a premalignant disease well known as monoclonal gammopathy of undetermined significance (MGUS) [2, 3]. MGUS affects $2 \%$ of the population above the age of 50 and it progresses to overt $\mathrm{MM}$ at a rate of $1 \%$ per year [4].

The biologic transition from normal plasma cells to MGUS and SMM to MM consists of many oncogenic events. An early event described in MGUS as well as MM is the dysregulation of a cyclin $D$ gene [5]. Secondary translocations, sometimes involving an Ig locus, can occur at any stage of plasma cell dyscrasia. Activating mutations of NRAS and KRAS are each present in about $15 \%$ of multiple myeloma tumors. Constitutive activation of the nuclear factor $\kappa \mathrm{B}(\mathrm{NF} \kappa \mathrm{B})$ pathway is mediated by mutations in some tumors during progression. In addition to these oncogenic events, the tumor cells are strongly dependent on the bone marrow microenvironment [6]. Substantial advances have been made in understanding the biology of MM through the study of the BM microenvironment. Indeed, the BM niche appears to play an important role in differentiation, migration, proliferation, survival, and drug resistance of the malignant plasma cells providing the preclinical evidences for targeting MM cells and bone marrow stromal cells (BMSC) as an antitumor strategy in this disease [7]. The cellular compartment is composed of hematopoietic cells and nonhematopoietic cells including fibroblasts/BMSC, endothelial cells (ECs), osteoclasts, and osteoblasts. The noncellular compartment is composed of the extracellular matrix (ECM) and the liquid milieu including cytokines, growth factors, and chemokines. MM cells home to the BM and adhere to ECM proteins and to BMSC. This trafficking (homing-egress) allows the progression or "metastasis" of the disease to new BM sites [7]. However the new host microenvironment is not well adapted to the cancer cells that metastasized into it, leading to the new concept of premetastatic niche [8]. Indeed significant changes occur in the microenvironment even before the first tumor cell homes 
to the bone marrow, as already described in solid tumor models [8]. In this paper, we discuss how BM may support $\mathrm{MM}$ cell growth and disease progression.

\section{The Bone Marrow Microenvironment}

\subsection{Cellular Compartment}

2.1.1. Bone Marrow Stromal Cells (BMSCs) in MM Progression. MM cells adhere to BMSC and ECM into the BM. Tumor cells bind to ECM proteins, such as type I collagen and fibronectin via syndecan 1 and very late antigen 4 (VLA-4) on MM cells and to BMSC VCAM-1 via VLA-4 on MM cells. Adhesion of tumor cells to BMSC activates many pathways resulting in upregulation of cell cycle regulating proteins and antiapoptotic proteins [9]. Specifically, the interaction between MM cells and BMSCs triggers NF$\kappa \mathrm{B}$ signaling pathway and interleukin-6 (IL-6) secretion in BMSCs. In turn, IL-6 enhances the production and secretion of VEGF by MM cells. The existence of this paracrine loop optimizes the BM milieu for MM tumor cell growth [10]. BMSC-MM cell interaction is also mediated through Notch. The interaction Notch-Notch ligand leads to activating Notch-signaling pathways both in MM cells as well as in BMSC, with induction of IL-6, vascular endothelial growth factor (VEGF), and insulin-like growth factor (IGF-1) secretion and is associated with MM cell proliferation and survival $[11,12]$. Moreover, BMSC from MM patients expresses several proangiogenic molecules, such as VEGF, basic-fibroblast growth factor (bFGF), angiopoietin 1 (Ang-1), transforming growth factor (TGF)- $\beta$, plateletderived growth factor (PDGF), hepatocyte growth factor (HGF), interleukin-1 (IL-1) [13]. Recently, BMSCs from MM patients have been shown to release exosomes, which are transferred to MM cells, thereby resulting in modulation of tumor growth in vivo, mediated by specific miRNA. This finding suggest that exosomes might constitute a novel mechanism for intercellular transfer of genetic information in the form of miRNA in clonal plasma cell disorders [14].

\subsubsection{Endothelial Cells and Angiogenesis in MM Progression.} $\mathrm{BM}$ angiogenesis represents a constant hallmark of MM progression, partly driven by release of pro-angiogenic cytokines from the tumor plasma cells, BMSC, and osteoclasts, such as VEGF, bFGF, and metalloproteinases (MMPs). Indeed, the adhesion between MM cells and BMSCs upregulates many cytokines with angiogenic activity, most notably VEGF and bFGF [15]. In MM cells, these pro-angiogenic factors may also be produced constitutively as a result of oncogene activation and/or genetic mutations [16]. Evidence for the importance of angiogenesis in the pathogenesis of MM was obtained from BM samples from MM patients [17]. The level of $\mathrm{BM}$ angiogenesis, as assessed by grading and/or microvessel density (MVD), is consistently increased in patients with active $\mathrm{MM}$ as compared to those with inactive disease or MGUS, a less advanced plasma cell disorder. Comparative gene expression profiling of multiple myeloma endothelial cells and MGUS endothelial cells has been performed in order to determine a genetic signature and to identify vascular mechanisms governing the malignant progression [18]. Twenty-two genes were found differentially expressed at relatively high stringency in MM endothelial cells compared with MGUS endothelial cells. Functional annotation revealed a role of these genes in the regulation of ECM formation and bone remodelling, cell adhesion, chemotaxis, angiogenesis, resistance to apoptosis, and cell-cycle regulation. The distinct endothelial cell gene expression profiles and vascular phenotypes detected in this study may influence remodelling of the bone marrow microenvironment in patients with active multiple myeloma. Overall, these evidences suggest that EC presents with functional, genetic, and morphologic features indicating their ability to induce $\mathrm{BM}$ neovascularization, resulting in $\mathrm{MM}$ cells growth, and disease progression, providing preclinical evidences for using antiangiogenic compounds in the treatment of MM.

2.1.3. Osteoclasts in MM Progression. The usual balance between bone resorption and new bone formation is lost in many cases of MM, resulting in bone destruction and the development of osteolytic lesions [19]. Bone destruction develops adjacent to MM cells, yet not in areas of normal bone marrow. There are several factors implicated in osteoclast activation, including receptor activator of NF- $\kappa \mathrm{B}$ ligand (RANKL), macrophage inflammatory protein-1a (MIP-1a), interleukin-3 (IL-3), and IL-6 [20]. RANK ligand is a member of the tumor necrosis factor (TNF) family and plays a major role in the increased osteoclastogenesis implicated in MM bone disease. RANK is a transmembrane signaling receptor expressed by osteoclast cells. MM cell binding to neighboring BMSC within the bone marrow results in increased RANKL expression. This leads to an increase in osteoclast activity through the binding of RANKL to its receptor, on osteoclast precursor cells, which further promotes their differentiation through NF- $\kappa \mathrm{B}$ and JunNterminal kinase pathway [21]. RANKL is also involved in inhibition of osteoclast apoptosis. Blocking RANKL with soluble form of RANK has been shown to modulate not only bone loss but also tumor burden in MM in vivo models [22]. Moreover osteoclasts constitutively secrete proangiogenic factors osteopontin that enhanced vascular tubule formation [23].

2.1.4. Osteoblasts in MM Progression. It has been reported that osteoblasts may contribute to MM pathogenesis by supporting MM cells growth and survival [24]. This could potentially result from the ability of osteoblasts to secrete IL-6 in coculture system with MM cells, thus increasing IL-6 levels within the BM milieu and therefore inducing MM plasma cells growth. Other mechanisms include the possible role of osteoblasts in stimulating MM cells survival by blocking TRAIL-mediated programmed MM cell death, by secreting osteoprotegerin (OPG), a receptor for both RANKL and TRAIL [25]. In addition, it is clear that suppression of osteoblast activity is responsible for both bone destructive process and progression of myeloma tumor burden. Several factors are responsible for suppression of osteoblast activity in MM such as DKK1 [26]. DKK1 is a Wnt-signaling antagonist secreted by MM cells and it 
inhibits osteoblast differentiation. DKK1 is significantly overexpressed in patients with MM who present with lytic bone lesions. Myeloma-derived DKK1 also disrupts Wnt-regulated OPG and RANKL production by osteoblasts. Studies have shown that blocking DKK1 and activating Wnt signaling prevents bone disease in MM but is also associated with a reduction in tumor burden [27-29].

\subsection{Noncellular Compartment}

2.2.1. Interleukin-6 in MM Progression. IL-6 is a key growth and survival factor in MM [30]. IL-6 is primarily produced by BMSC and osteoblasts and mediates paracrine MM cell growth and is also secreted by MM tumor cells in an autocrine manner [31]. IL-6 secretion from BMSC is upregulated by many molecules/cytokines (i.e., CD40, TNF$\alpha$, VEGF, IL- $1 \beta$, TGF- $\beta$ ) and MM cell adherence. IL- $1 \beta$ appears to be one of the major cytokines responsible for the paracrine production of IL- 6 by the BMSC. The aberrant production of IL- $1 \beta$ by the MM cells induces IL- 6 production by BMSC, which in turn supports the growth and survival of the myeloma cells [32]. Importantly, NF- $\kappa \mathrm{B}$ plays a central role in cytokine- and adhesion-mediated IL- 6 upregulation, and specific inhibition of NF- $\kappa$ B blocks IL-6 secretion [33]. After binding with its receptor, IL-6 triggers activation of MEK/MAPK, JAK/STAT3, and PI3K/Akt signaling pathways [34]. IL-6 induces proliferation of the tumor plasma cells by activating the RAS/Raf/MEK-ERK signaling pathway. IL6 is also able to inhibit the antiproliferation effects of cyclin-dependent kinase (CDK) inhibitors p21 and p27 through the PI3K/Akt pathway [31]. IL-6 activation of the JAK/STAT3 pathway induces tumor cells survival by upregulation/activation of anti-apoptotic proteins Mcl-1 and Bcl- $\mathrm{X}_{\mathrm{L}}$ and c-Myc. Clinically, elevated serum IL-6 levels are associated with a poor prognosis and reflect the proliferation fraction of MM cells within patients [35]. Otherwise IL-1 receptor targeted therapies have shown activity in increasing PFS of patients with smoldering disease [36].

2.2.2. Insulin-Like Growth Factor 1 in MM Progression. Insulin-like growth factor 1 (IGF-1) is involved in tumorigenesis of several solid cancers [37]. In MM, IGF-1 is secreted by the BMSC and osteoblasts and induces growth, survival, and migration of MM cells. The phosphorylation of IGF-1 receptor (IGF-1R), after IGF-1 binding, leads to activation of MAPK and PI3K/Akt signaling pathway [38]. Activation of Akt leads to activating the anti-apoptotic proteins Bcl$\mathrm{X}_{\mathrm{L}}, \mathrm{Bcl}-2$ and downregulating the proapoptotic protein Bim, thereby promoting cell survival. IGF-1 is also well known for its metabolic effects. Interestingly, IGF-1 could be involved in the pathophysiology that relates obesity and diabetes to neoplasia [37].

2.2.3. Vascular Endothelial Growth Factor (VEGF) in MM Progression. VEGF represents a well-known proangiogenic factor: its levels increase in several hematologic malignancies including MM [15]. In MM, VEGF is produced both by MM cells and BMSC, and its secretion is stimulated by different cytokines and cell growth factors such as IL-6, bFGF, TGF$\beta$ or tumor necrosis factor (TNF)- $\alpha$. It is an important factor in the formation of new vasculature; upon binding with VEGF receptor-1 and -2 (VEGFR-1 and VEGFR-2), it triggers proliferation, migration, differentiation, and survival of BMSC and EC, through several signaling pathway such as Ras GAP, FAK, PI3K/Akt, MEK/ERK, and STAT [15]. Blood vessels are required for tumor growth and progression for provision of vital oxygen and nutrients. It has been shown that increased microvessel density (MVD) in BM of MM patients is associated with poor prognosis.

\section{Homing and Egress}

The initiation of MM is likely from long-lived plasma cells that develop in germinal center of lymphoid tissues and home to the BM. Oncogenic events along with support of the microenvironment allow the growth, survival, and proliferation of these cells in the initial sites of the BM niche [7]. Furthermore, some studies showed the presence of a small number of circulating plasma cells in MM and its association with a poor prognosis [39]. Migration of cells through the blood to the bone marrow niches requires active navigation, a process termed homing. The first step in the homing process is the rolling of the MM cell along the EC through selectin. The adhesion and extravasation are induced by activation of integrin-expressed by MM cell-such as LFA-1 and VLA-4 [40]. The SDF-1/CXCR4 axis plays a critical role in homing of MM cells to the BM. Studies to identify expression of chemokine receptors in MM have shown large variations in CXCR4 expression ranging from 10 to $100 \%$. SDF-1 induces migration of MM cells in vitro and homing into the bone marrow in vivo. Moreover, CXCR4 knockdown led to significant inhibition of migration to SDF-1 in MM cell lines and primary CD138+ cells [41]. $\mathrm{MM}$ is characterized by the disseminated involvement of the $\mathrm{BM}$, and its progression involves a continuous circulation of the MM cells in the peripheral blood and homing back to the BM. Mobilization or egress of cells out of the bone marrow could be enhanced by disrupting the SDF1/CXCR4 axis. This may occur by decreasing SDF-1 by protease in the BM milieu [42], or by upregulation of CXCR4 expression by hypoxia. Indeed, the bone marrow niche is quite hypoxic $\left(1 \%-2 \% \mathrm{O}_{2}\right)$ [43]. It has also been shown that hypoxia leads to inactivation of E-cadherin and activation of transcription factors regulating epithelial-mesenchymal transition, including Snail and Twist, indicating that this mechanism can participate to the egress process.

\section{Premetastatic Niche}

Although preparation of the premetastatic niche has not been studied in MM, several works have shown the importance of the premetastatic niche in solid cancer metastasis to the bone marrow. Indeed, the new host microenvironment is not well adapted to the cancer cells that metastasized into it [8]. Therefore, significant changes in the stroma, endothelial cells, ECM constituents, cytokines, and chemokines need 
to occur to allow for the growth and survival of these metastastic cells. Preparation of the metastatic niche occurs even before the first metastastic cell arrives. Evidence has emerged that growth factors and cytokines secreted by the tumor prepare tissues for tumor cell engraftment [44]. For example, bone marrow-derived hematopoietic cells that express VEGFR1 as the fibronectin receptor VLA-4 are localized to the premetastatic sites before the arrival of tumor cells [44]. Moreover, microvesicles such as exosomes have been shown to alter the premetastatic niche in different studies [45]. Exosomes are small vesicles (30-100 nm) of endocytic origin, which are released in the extracellular milieu by several cell types. Previous studies have shown the intriguing role of exosomes in tumor progression. Recently, melanomaderived exosomes have been shown to induce neoangiogenesis at pre-metastatic niche sites. RAB1A, RAB5B, RAB7, and RAB27A, regulators of membrane trafficking and exosome formation, are highly expressed in melanoma cells. Rab27A RNA interference decreased exosome production, preventing bone marrow education and reducing, tumor growth and metastasis. These data show that exosome production, transfer and education of bone marrow cells support tumor growth and progression to the bone marrow [46].

\section{Conclusion}

Several reports have clearly indicated that MM pathophysiology is supported by a strong interaction between the clonal plasma cells and the surrounding bone marrow microenvironment; indeed, there are several autocrine or paracrine circuits of growth that support the transformation from an MGUS stage to an active MM stage. By understanding the interaction occurring between BMSC and MM cells, and vice versa, we have now available the preclinical rational for testing novel therapeutical approaches in order to better target not only the MM cell clone, but also the BM milieu, thus preventing $\mathrm{MM}$ disease progression.

\section{References}

[1] R. A. Kyle and S. V. Rajkumar, "Criteria for diagnosis, staging, risk stratification and response assessment of multiple Myeloma," Leukemia, vol. 23, no. 1, pp. 3-9, 2009.

[2] O. Landgren, R. A. Kyle, R. M. Pfeiffer et al., "Monoclonal gammopathy of undetermined significance (MGUS) consistently precedes multiple Myeloma: a prospective study," Blood, vol. 113, no. 22, pp. 5412-5417, 2009.

[3] B. M. Weiss, J. Abadie, P. Verma, R. S. Howard, and W. M. Kuehl, "A monoclonal gammopathy precedes multiple Myeloma in most patients," Blood, vol. 113, no. 22, pp. 5418-5422, 2009.

[4] R. A. Kyle, B. G. Durie, S. V. Rajkumar et al., "Monoclonal gammopathy of undetermined significance (MGUS) and smoldering (asymptomatic) multiple Myeloma: IMWG consensus perspectives risk factors for progression and guidelines for monitoring and management," Leukemia, vol. 24, no. 6, pp. 1121-1127, 2010.

[5] P. L. Bergsagel, W. M. Kuehl, F. Zhan, J. Sawyer, B. Barlogie, and J. Shaughnessy Jr., "Cyclin D dysregulation: an early and unifying pathogenic event in multiple Myeloma," Blood, vol. 106, no. 1, pp. 296-303, 2005.

[6] N. Korde, S. Y. Kristinsson, and O. Landgren, "Monoclonal gammopathy of undetermined significance (MGUS) and smoldering multiple Myeloma (SMM): novel biological insights and development of early treatment strategies," Blood, vol. 117, no. 21, pp. 5573-5581, 2011.

[7] I. M. Ghobrial, "Myeloma as a model for the process of metastasis: implications for therapy," Blood, vol. 120, no. 1, pp. 2030, 2012.

[8] I. J. Fidler, "The pathogenesis of cancer metastasis: the "seed and soil" hypothesis revisited," Nature Reviews Cancer, vol. 3, no. 6, pp. 453-458, 2003.

[9] T. Hideshima, P. L. Bergsagel, W. M. Kuehl, and K. C. Anderson, "Advances in biology of multiple Myeloma: clinical applications," Blood, vol. 104, no. 3, pp. 607-618, 2004.

[10] S. Kumar, T. E. Witzig, M. Timm et al., "Expression of VEGF and its receptors by Myeloma cells," Leukemia, vol. 17, no. 10, pp. 2025-2031, 2003.

[11] F. Radtke and K. Raj, "The role of Notch in tumorigenesis: oncogene or tumour suppressor," Nature Reviews Cancer, vol. 3, no. 10, pp. 756-767, 2003.

[12] Y. Nefedova, P. Cheng, M. Alsina, W. S. Dalton, and D. I. Gabrilovich, "Involvement of Notch-1 signaling in bone marrow stroma-mediated de novo drug resistance of Myeloma and other malignant lymphoid cell lines," Blood, vol. 103, no. 9, pp. 3503-3510, 2004.

[13] N. Giuliani, P. Storti, M. Bolzoni, B. D. Palma, and S. Bonomini, "Angiogenesis and multiple Myeloma," Cancer Microenvironment, vol. 4, no. 3, pp. 325-337, 2011.

[14] A. M. Roccaro, A. Sacco, A. K. Azab et al., "Stroma-derived exosomes mediate oncogenesis in multiple Myeloma," Blood, vol. 118, abstract 625, 2011, ASH Annual Meeting Abstracts.

[15] K. Podar and K. C. Anderson, "The pathophysiologic role of VEGF in hematologic malignancies: therapeutic implications," Blood, vol. 105, no. 4, pp. 1383-1395, 2005.

[16] S. V. Rajkumar and T. E. Witzig, "A review of angiogenesis and antiangiogenic therapy with thalidomide in multiple Myeloma," Cancer Treatment Reviews, vol. 26, no. 5, pp. 351-362, 2000.

[17] S. Kumar, M. A. Gertz, A. Dispenzieri et al., "Prognostic value of bone marrow angiogenesis in patients with multiple Myeloma undergoing high-dose therapy," Bone Marrow Transplantation, vol. 34, no. 3, pp. 235-239, 2004.

[18] R. Ria, K. Todoerti, S. Berardi et al., "Gene expression profiling of bone marrow endothelial cells in patients with multiple Myeloma," Clinical Cancer Research, vol. 15, no. 17, pp. 53695378, 2009.

[19] R. Bataille, D. Chappard, C. Marcelli et al., "Mechanisms of bone destruction in multiple Myeloma: the importance of an unbalanced process in determining the severity of lytic bone disease," Journal of Clinical Oncology, vol. 7, no. 12, pp. 19091914, 1989.

[20] G. D. Roodman, "Pathogenesis of Myeloma bone disease," Leukemia, vol. 23, no. 3, pp. 435-441, 2009.

[21] L. A. Ehrlich and G. D. Roodman, "The role of immune cells and inflammatory cytokines in Paget's disease and multiple Myeloma," Immunological Reviews, vol. 208, pp. 252-266, 2005.

[22] S. Yaccoby, R. N. Pearse, C. L. Johnson, B. Barlogie, Y. Choi, and J. Epstein, "Myeloma interacts with the bone marrow microenvironment to induce osteoclastogenesis and is dependent on osteoclast activity," British Journal of Haematology, vol. 116, no. 2, pp. 278-290, 2002. 
[23] Y. Tanaka, M. Abe, M. Hiasa et al., "Myeloma cell-osteoclast interaction enhances angiogenesis together with bone resorption: a role for vascular endothelial cell growth factor and osteopontin," Clinical Cancer Research, vol. 13, no. 3, pp. 816-823, 2007.

[24] A. Karadag, B. O. Oyajobi, J. F. Apperley, R. Graham, G. Russell, and P. I. Croucher, "Human Myeloma cells promote the production of interleukin 6 by primary human osteoblasts," British Journal of Haematology, vol. 108, no. 2, pp. 383-390, 2000.

[25] C. M. Shipman and P. I. Croucher, "Osteoprotegerin is a soluble decoy receptor for tumor necrosis factor-related apoptosis-inducing ligand/Apo2 ligand and can function as a paracrine survival factor for human Myeloma cells," Cancer Research, vol. 63, no. 5, pp. 912-916, 2003.

[26] E. Tian, F. Zhan, R. Walker et al., "The role of the Wntsignaling antagonist DKK1 in the development of osteolytic lesions in multiple Myeloma," The New England Journal of Medicine, vol. 349, no. 26, pp. 2483-2494, 2003.

[27] S. Yaccoby, W. Ling, F. Zhan, R. Walker, B. Barlogie, and J. D. Shaughnessy Jr., "Antibody-based inhibition of DKK1 suppresses tumor-induced bone resorption and multiple Myeloma growth in vivo," Blood, vol. 109, no. 5, pp. 21062111, 2007.

[28] C. M. Edwards, J. R. Edwards, S. T. Lwin et al., "Increasing wnt signaling in the bone marrow microenvironment inhibits the development of Myeloma bone disease and reduces tumor burden in bone in vivo," Blood, vol. 111, no. 5, pp. 2833-2842, 2008.

[29] M. Fulciniti, P. Tassone, T. Hideshima et al., "Anti-DKK1 $\mathrm{mAb}(\mathrm{BHQ} 880)$ as a potential therapeutic agent for multiple Myeloma," Blood, vol. 114, no. 2, pp. 371-379, 2009.

[30] M. Kawano, T. Hirano, T. Matsuda et al., "Autocrine generation and requirement of BSF-2/IL-6 for human multiple Myelomas," Nature, vol. 332, no. 6159, pp. 83-85, 1988.

[31] T. Hideshima, N. Nakamura, D. Chauhan, and K. C. Anderson, "Biologic sequelae of interleukin-6 induced PI3-K/Akt signaling in multiple Myeloma," Oncogene, vol. 20, no. 42, pp. 5991-6000, 2001.

[32] C. A. Dinarello, "Interleukin-1 in the pathogenesis and treatment of inflammatory diseases," Blood, vol. 117, no. 14, pp. 3720-3732, 2011.

[33] D. Chauhan, S. Kharbanda, A. Ogata et al., "Interleukin-6 inhibits Fas-induced apoptosis and stress-activated protein kinase activation in multiple Myeloma cells," Blood, vol. 89, no. 1, pp. 227-234, 1997.

[34] T. Hideshima, C. Mitsiades, G. Tonon, P. G. Richardson, and K. C. Anderson, "Understanding multiple Myeloma pathogenesis in the bone marrow to identify new therapeutic targets," Nature Reviews Cancer, vol. 7, no. 8, pp. 585-598, 2007.

[35] H. Ludwig, D. M. Nachbaur, E. Fritz, M. Krainer, and H. Huber, "Interleukin-6 is a prognostic factor in multiple Myeloma," Blood, vol. 77, no. 12, pp. 2794-2795, 1991.

[36] J. A. Lust, M. Q. Lacy, S. R. Zeldenrust et al., "Induction of a chronic disease state in patients with smoldering or indolent multiple Myeloma by targeting interleukin $1 \beta$-induced interleukin 6 production and the Myeloma proliferative component," Mayo Clinic Proceedings, vol. 84, no. 2, pp. 114122, 2009.

[37] M. Pollak, "Insulin and insulin-like growth factor signalling in neoplasia," Nature Reviews Cancer, vol. 8, no. 12, pp. 915-928, 2008.

[38] E. De Bruyne, T. J. Bos, F. Schuit et al., "IGF-1 suppresses Bim expression in multiple Myeloma via epigenetic and posttranslational mechanisms," Blood, vol. 115, no. 12, pp. 2430-2440, 2010.

[39] G. S. Nowakowski, T. E. Witzig, D. Dingli et al., "Circulating plasma cells detected by flow cytometry as a predictor of survival in 302 patients with newly diagnosed multiple Myeloma," Blood, vol. 106, no. 7, pp. 2276-2279, 2005.

[40] F. Sanz-Rodríguez, A. Hidalgo, and J. Teixidó, "Chemokine stromal cell-derived factor- $1 \alpha$ modulates VLA- 4 integrinmediated multiple Myeloma cell adhesion to CS-1/fibronectin and VCAM-1," Blood, vol. 97, no. 2, pp. 346-351, 2001.

[41] Y. Alsayed, H. Ngo, J. Runnels et al., "Mechanisms of regulation of CXCR4/SDF-1 (CXCL12)-dependent migration and homing in multiple Myeloma," Blood, vol. 109, no. 7, pp. 2708-2717, 2007.

[42] T. Lapidot, "Mechanism of human stem cell migration and repopulation of NOD/SCID and B2mnull NOD/SCID mice: the role of SDF-1/CXCR4 interactions," Annals of the New York Academy of Sciences, vol. 938, pp. 83-95, 2001.

[43] M. G. Cipolleschi, P. D. Sbarba, and M. Olivotto, "The role of hypoxia in the maintenance of hematopoietic stem cells," Blood, vol. 82, no. 7, pp. 2031-2037, 1993.

[44] R. N. Kaplan, R. D. Riba, S. Zacharoulis et al., "VEGFR1positive haematopoietic bone marrow progenitors initiate the pre-metastatic niche," Nature, vol. 438, no. 7069, pp. 820-827, 2005.

[45] B. Psaila and D. Lyden, "The metastatic niche: adapting the foreign soil," Nature Reviews Cancer, vol. 9, no. 4, pp. 285-293, 2009.

[46] H. Peinado, M. Aleckovic, S. Lavotshkin et al., "Melanoma exosomes educate bone marrow progenitor cells toward a prometastatic phenotype through MET," Nature Medicine, vol. 18, no. 6, pp. 883-891, 2012. 


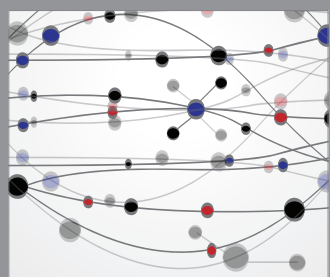

The Scientific World Journal
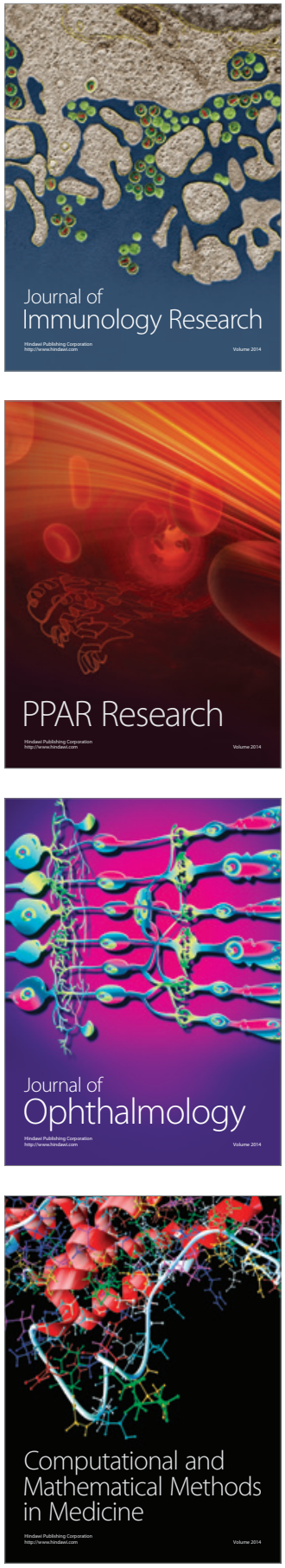

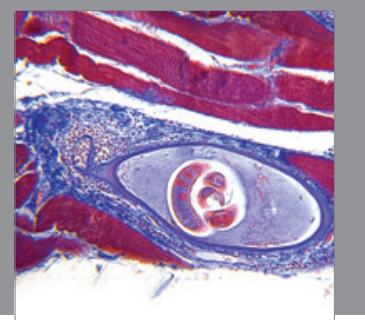

Gastroenterology

Research and Practice
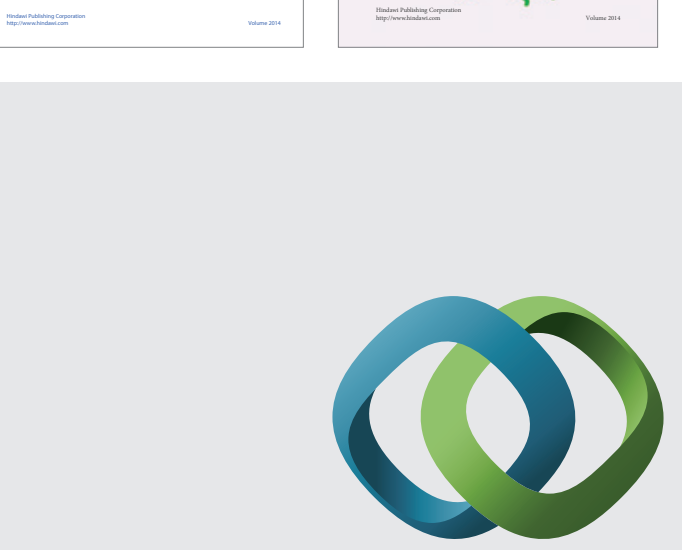

\section{Hindawi}

Submit your manuscripts at

http://www.hindawi.com
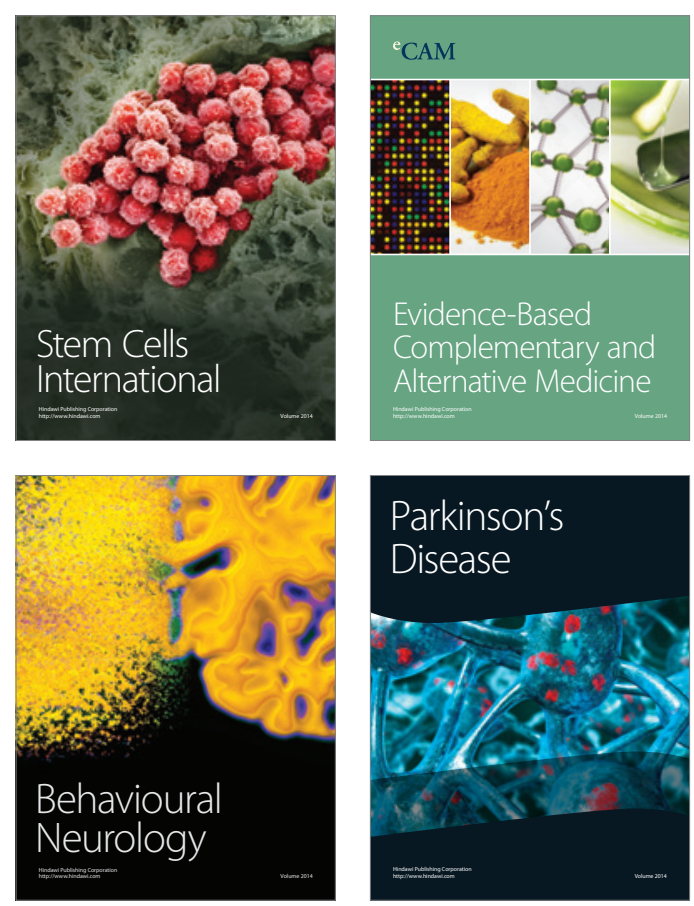

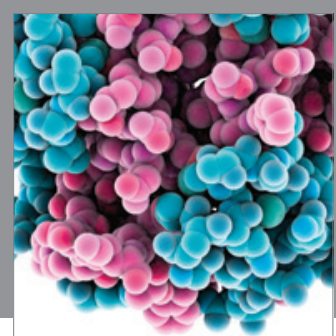

Journal of
Diabetes Research

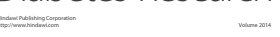

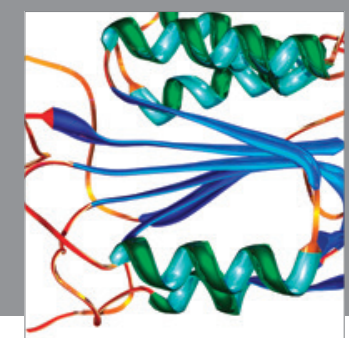

Disease Markers
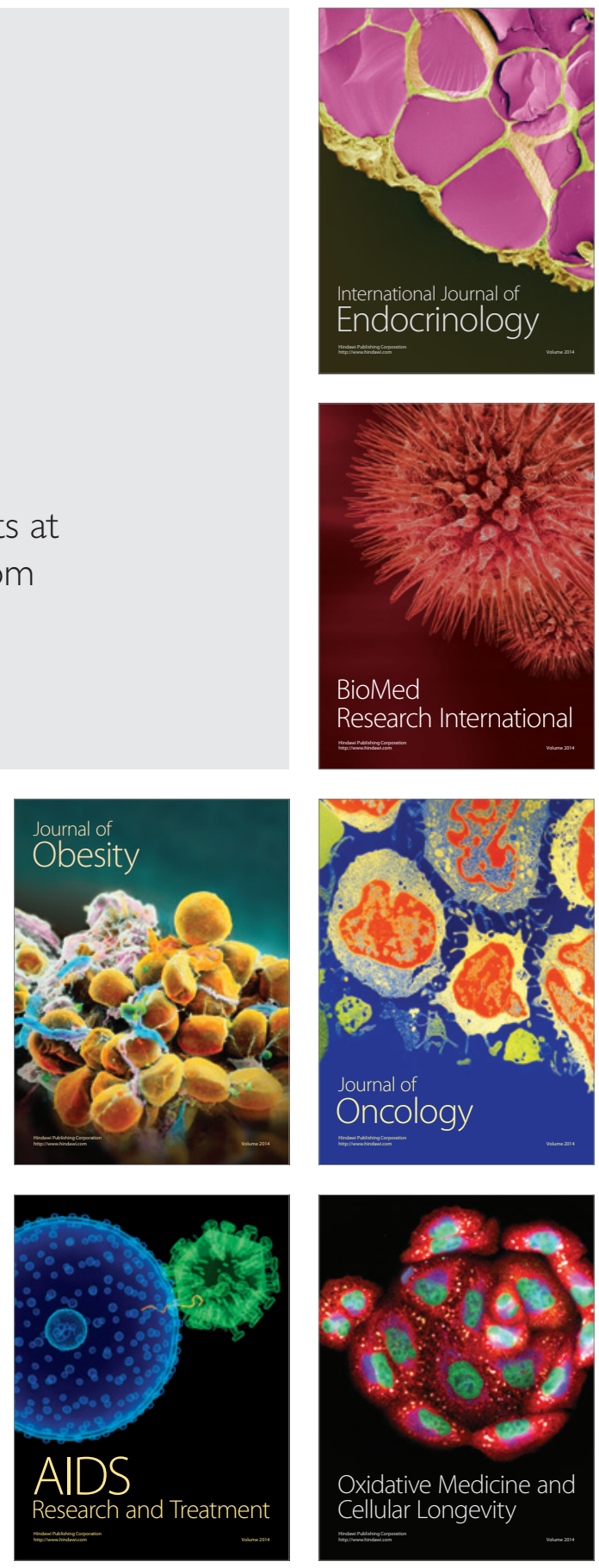